\title{
Are Patients Getting Drunk on Surgical Preparation? Absorption of Isopropyl Alcohol Across Vaginal Mucosa
}

\author{
Brittany Deller ${ }^{1}$, Craig Railton ${ }^{2}$ and Jacob McGee ${ }^{1 *}$ \\ ${ }^{1}$ Department of Obstetrics and Gynecology, Canada \\ ${ }^{2}$ Department of Clinical Pharmacology and Department of Anesthesia and Perioperative Medicine, Canada \\ *Corresponding author: Jacob McGee, Department of Obstetrics and Gynecology, Canada
}

\section{ARTICLE INFO}

Received: 幽 September 02, 2019

Published: 慧 September 10, 2019

Citation: Brittany Deller, Craig Railton, Jacob McGee. Are Patients Getting Drunk on Surgical Preparation? Absorption of Isopropyl Alcohol Across Vaginal Mucosa. Biomed J Sci \& Tech Res 21(2)-2019. BJSTR. MS.ID.003576.

\section{ABSTRACT}

Objective: To determine vaginal absorption of isopropyl alcohol solution in patients prepped vaginally with a solution of $2 \mathrm{v} / \mathrm{w}$ chlorhexidine and $70 \%$ isopropyl alcohol.

Methods: The amount of vaginal prep was quantified by weighing sponges prior to and after vaginal preparation. A volatile screen quantifying absorption of four known toxins including isopropyl alcohol was conducted at 6 time points - prior to vaginal preparation (baseline), and at 5, 10, 15, 30, and 45 minutes post application. REB approval was obtained for 10 patients; however, the study was stopped after collecting samples on 3 patients to mitigate both patient exposure to blood tests and study cost.

Results: Three patients completed the full protocol with all samples collected. Average possible isopropanol dose was $20 \mathrm{ml}$, recognizing that actual dose was smaller due to spillage onto the external genitalia. The expected absorbed level of isopropyl alcohol ranged across the three patients from $3.17 \mathrm{mmol} / \mathrm{L}$ to $8.34 \mathrm{mmol} / \mathrm{L}$. None of the toxicology screens conducted registered an increase above the baseline level of $<1.0$ $\mathrm{mmol} / \mathrm{L}$ isopropyl alcohol.

Conclusion: Our study shows that isopropyl alcohol absorption is not a concern when solutions containing isopropyl alcohol are used to prepare the vagina for surgery. While absorption of the alcohol is likely occurring, it is being metabolized at a rate at least equal to that absorption, rendering it undetectable in the peripheral blood.

\section{Introduction}

Individuals will sometimes go to great lengths to become intoxicated. As an example, a trend noted in popular media is the use of alcohol-soaked tampons as an effective means of becoming intoxicated [1,2]. The senior author first read about this phenomenon while living in Bergen Norway, where some enterprising adolescents had maximized the effect of a limited supply by soaking tampons in ethanol to thoroughly inebriate themselves. Many years later, while working as a gynecologic oncologist in a tertiary care setting, I learned that several colleagues as part of antisepsis prepared the vagina prior to surgery with a $70 \%$ isopropyl alcohol chlorhexidine solution. While debating a colleague in departmental grand rounds, I was unable to convince him that $70 \%$ isopropyl alcohol is relatively contraindicated on a mucosal membrane because of its obvious irritative effects. Upping the ante, I recalled my time in Norway, querying if isopropyl alcohol, applied to the vagina as part of the surgical preparation, could in fact be contributing to low level intoxication and adverse peri-operative outcomes.

The dense meshwork of blood vessels and a permeable mucosa makes the vagina an ideal route of administration for many other low molecular weight drugs $[3,4]$. Substances absorbed vaginally bypass first pass metabolism thereby increasing the bioavailability of the absorbed drugs [3,5]. Although isopropyl alcohol exposure is usually through oral ingestion it has also been reported to be 
absorbed by inhalation, dermally and rectally [6,7]. Isopropyl alcohol acts as a CNS depressant and with large doses orally it can cause respiratory depression and hypotension and is an irritant to the mucosa potentially causing hematemesis and haemorrhagic gastritis [7,8]. Other symptoms described following ingestion include hypothermia, sinus tachycardia and renal tubular necrosis [8]. The most common effects of isopropyl alcohol at subtoxic doses are an increased osmolar gap, ketonemia and ketouria [7]. Absorbing isopropyl alcohol into the bloodstream may have potential implications on anesthesia and post- operative recovery. Isopropyl is a sedative-hypnotic with a toxicity that parallels that of ethanol as they share strong structural similarity. In terms of CNS depression isopropyl alcohol is thought to be more potent than ethanol $[8,9]$. This study was undertaken to assess if $2 \% \mathrm{w} / \mathrm{v}$ chlorhexidine gluconate with $70 \% \mathrm{v} / \mathrm{v}$ isopropyl alcohol when used vaginally for surgical preparation is absorbed across the vaginal mucosa into the blood stream of patients.

\section{Materials and Methods}

The research protocol was approved by the Research Ethics Board at Western University and the Lawson Research Institute. REB approval was obtained for 10 patients. Patients were consented in the gynecologic oncology clinic. The vaginal preparation sponges containing 70\% isopropyl alcohol were weighed prior to and after application in the operating theatre. The weighted difference was used to determine the isopropyl alcohol dose. A volatile screen quantifying absorption of four known toxins including isopropyl alcohol was conducted at 6 time points - prior to vaginal preparation (baseline), and at 5, 10, 15, 30, and 45 minutes post application. The volatile screen is a gas liquid chromatography test capable of detecting isopropyl alcohol at $1 \mathrm{mmol} / \mathrm{L}$. Samples were sent immediately to lab for evaluation.

Inclusion criteria were gynecologic oncology surgical patients scheduled for procedures with duration over 90mins (to allow time for serial blood draws) receiving $2 \% \mathrm{w} / \mathrm{v}$ chlorhexidine gluconate with $70 \% \mathrm{v} / \mathrm{v}$ isopropyl alcohol vaginally as surgical preparation. There were no exclusion criteria. Descriptive statistics were employed in the analysis of the data. The study is reported in accordance with the STROBE guidelines for observational studies. The authors have read and understood the BMJ Group policy on declaration of interests and declare no competing interests. This study was self-funded.

\section{Results}

A total of three patients completed the full protocol with all samples collected (Table 1). The theoretical isopropyl alcohol dose ranged from $3.17-8.34 \mathrm{mmol} / \mathrm{L}$. We calculated a theoretical dose of $25 \mathrm{~mL}$ prep in a $70 \mathrm{~kg}$ woman to be $6.4 \mathrm{mmol} / \mathrm{L}$. The serum isopropyl alcohol level from the volatile test was 0 at each time point for all three patients (Figure 1). The investigators chose to stop the study early due to the arising trend to minimize patient exposure to unnecessary testing and limit study cost.

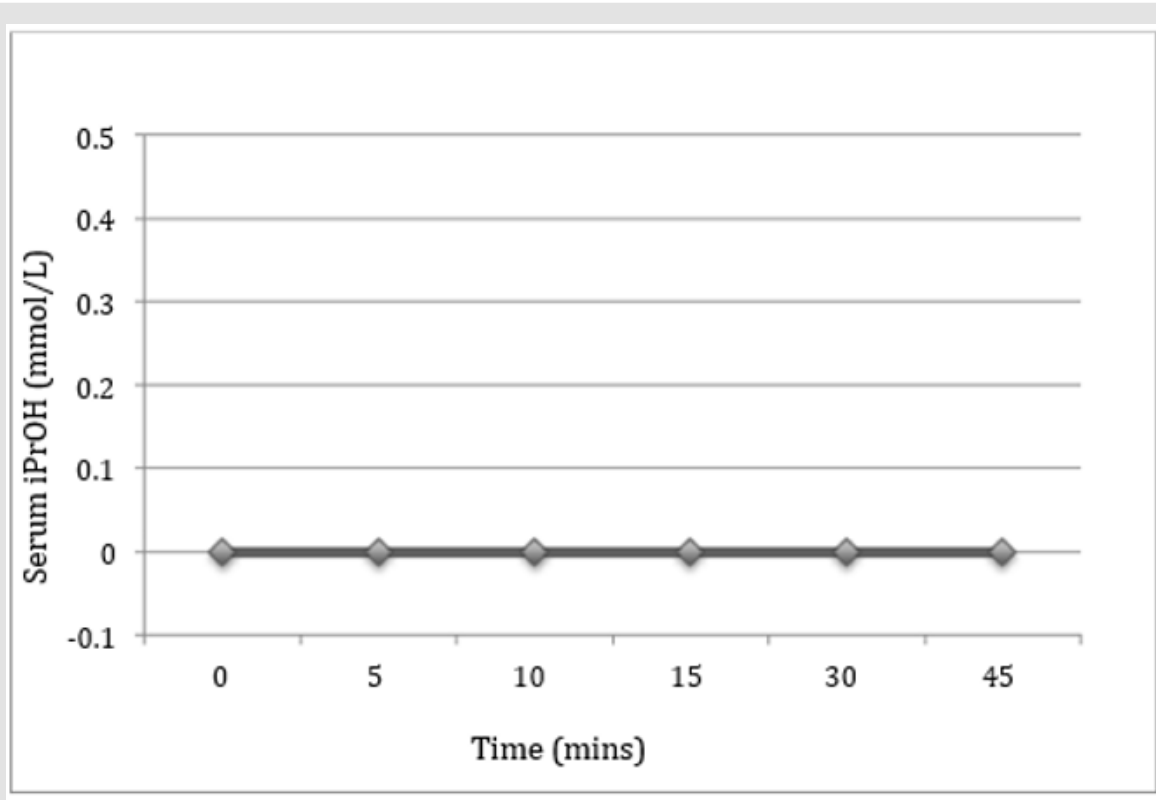

Figure 1: Average measured serum isopropyl alcohol (iPrOH) level (mmol/L).

Table 1: Calculated estimated plasma concentration of Isopropyl alcohol (iPrOH) assuming optimal conditions. Calculations with $\mathrm{iPrOH}$ molecular weight 60.09 ; volume of distribution $0.65 \mathrm{~L} / \mathrm{kg}$ and bioavailability $100 \%$.

\begin{tabular}{|c|c|c|c|c|c|c|c|c|}
\hline Patient & Weight & Dose in mL & iPrOH dose in g & Moles iPrOH & Est. Vd (L) & Est. [iPrOH] & [iPrOH] mmol & [iPrOH] mg/L \\
\hline 1 & 53.7 & 25 & 17.5 & 0.29122 & 34.9 & 0.00834 & 8.34 & 502 \\
\hline 2 & 82.3 & 15 & 10.5 & 0.174 & 53.8 & 0.00325 & 3.25 & 195 \\
\hline
\end{tabular}




\begin{tabular}{|c|c|c|c|c|c|c|c|c|}
\hline 3 & 113.1 & 20 & 14 & 0.233 & 73.5 & 0.00317 & 3.17 & 191 \\
\hline $\begin{array}{c}\text { Average } \\
70 \text { kg Pt. }\end{array}$ & 70 & 25 & 17.5 & 0.29122 & 45.5 & 0.00640 & 6.40 \\
\hline
\end{tabular}

$\mathrm{iPrOH}$ - Isopropyl alcohol

Est. Vd - Estimate volume distribution

\section{Discussion}

Our study shows that isopropyl alcohol is not absorbed at a measurable level when patients are prepped vaginally with $2 \% \mathrm{w} / \mathrm{v}$ chlorhexidine gluconate with $70 \% \mathrm{v} / \mathrm{v}$ isopropyl alcohol prior to surgery. Isopropyl alcohol is a toxic substance producing adverse physiologic effects at doses as low as $0.5 \mathrm{~mL}$ per $\mathrm{kg}$ ( $3.32 \mathrm{mmol} / \mathrm{L})$. Toxicity occurs at doses of $1 \mathrm{~mL}$ per $\mathrm{kg}(6.64 \mathrm{mmol} / \mathrm{L})$ and lethal doses occur between $2-4 \mathrm{~mL}$ per $\mathrm{kg}$ (23 to $46 \mathrm{mmol} / \mathrm{L}$ ) [10]. It would be theoretically possible to deliver a toxic dose in the preparation of a surgical site if the right conditions were met sufficient volume of an isopropyl alcohol prep and extended exposure on an absorptive membrane. Up to 100 percent of the dose of isopropyl alcohol can be adsorbed from a mucous membrane or skin if continuous contact is maintained between the surface and the isopropyl alcohol for up to two hours [10]. Assuming optimal conditions for absorption of the isopropyl alcohol are met, the estimated plasma concentrations of isopropyl alcohol were calculated using patient weight and dose administered in the vagina and shown in Table 1. Most patients could receive a dose that would cause adverse physiologic effects ( $>3.32 \mathrm{mmol} / \mathrm{L}$ ) and smaller patients could easily receive a toxic dose ( $>6.64 \mathrm{mmol} / \mathrm{L})$ as seen in patient 1 from Table 1 .

Many factors influence the absorption across the vaginal mucosa including thickness of the vaginal epithelium that changes cyclically, vaginal secretions and vaginal enzymes [4]. Absorption of drugs through the vagina can be challenging because of the thickness of the vaginal wall and the tight junctions between the cells. For a substance to be absorbed vaginally it must be fat soluble and have prolonged contact with the vaginal wall. Isopropyl alcohol is very fat and is used to enhance the absorption of other less soluble drugs though skin or a mucous membrane in many drug delivery devices [3]. Isopropyl alcohol follows first order kinetics with a half-life of 2-8.5 hours, reaching peak plasma concentration when absorbed orally at 30 minutes [7]. The longer the hydrocarbon chain, the more soluble an alcohol is, suggesting that isopropyl alcohol is more readily absorbed vaginally than is ethanol [11].

A plausible explanation for why we did not observe isopropyl alcohol in the peripheral blood is that the isopropyl alcohol did not have sustained contacted with the vagina secondary to leakage from the vagina. Secondly, the estimated dose in our study likely exceeds the actual dose delivered to the vaginal mucosa as spillage onto the external genitalia occurred in each case as the sponge passed through the labia into the vagina during the surgical preparation. Finally, while absorption of some of the alcohol may have occurred, we speculate that it was metabolized at a rate at least equal to that of absorption, rendering it undetectable in the peripheral blood. Isopropyl alcohol is metabolized in the liver by alcohol dehydrogenase to acetone [7]. Future research on this topic could use a baseline acetone level and serial measurements of acetone to quantify if the isopropyl alcohol is indeed being absorbed but being metabolized rapidly. It may also beneficial to calculate the osmolar gap and serum ketones at different time points as isopropyl alcohol can increase the osmolar gap and cause ketonemia [7]. These initiatives would represent a completely academic exercise and we will not be undertaking this simply to prove our point to a colleague.

Chlorhexidine has been shown to have greater antimicrobial effects in the vagina over povidone-iodine2. While chlorhexidine with $70 \%$ isopropyl alcohol is used by some at our institution, the American Congress of Obstetricians and Gynecologist recommends against the use of isopropyl alcohol at high concentration in the vagina because of irritation [12]. Chlorhexadine containing solutions with lower isopropyl alcohol concentrations are available. Our study was able to show that use of a chlorhexidine preparation with $70 \%$ isopropyl alcohol should not be halted out of concern about vaginal absorption into the bloodstream resulting in adverse physiological effects. While intoxication from 70\% isopropyl alcohol is not a concern when the vagina is prepped in a conventional fashion, we still do not recommend usage of high concentrations on a mucous membrane like the vagina. While future research is not required, common sense when selecting an appropriate solution for vaginal preparation is.

\section{Conflict of Interest}

The authors have no identified potential conflicts of interest of a financial or other nature.

\section{References}

1. Stogner JM, Eassey JM, Balwin JM, Miller BL (2014) Innovative alcohol use: assessing the prevalence of alcohol without liquid and other nonoral routes of alcohol administration. Drug Alcohol Depend 142: 74-78.

2. Vorherr H, Vorherr UF, Mehta P, Ulrich JA, Messer RH (1984) Antimicrobial effect of chlorhexidine and povidone-iodine on vaginal bacteria Infect 8(3): 195-199.

3. Hussain A, Ahsan F (2005) The vagina as a route for systemic drug delivery. J Control Release 103(2): 301-313.

4. Srikrishna S, Cardozo L (2013) The vagina as a route for drug delivery. Int Urogynecol J 24(4): 537-543.

5. Justin-Temu M, Damian F, Kinget R, Van Den Mooter G (2004) Intravaginal gels as drug delivery systems. J Womens Health (Larchmt) 13(7): 834844 .

6. Barnett JM, Plotnick M, Fine KC (1990) Intoxication after an isopropyl alcohol enema. Ann Intern Med 133(8): 638-639.

7. Slaughter RJ, Mason RW, Beasley DM, Vale JA, Schep LJ (2014) Isopropanol poisoning. Clin Toxicol (Phila) 52(5): 470-478. 
8. Vale A (2007) Isopropanol. Medicine 35(12): 626.

9. Trummel J, Ford M, Austin P (1996) Ingestion of an unknown alcohol. Ann Emerg Med 27(3): 368-374.

10. InCHEM. Isopropyl Alcohol.

11. Hwang S, Owada E, Yotsuyanagi T, Suhardja L, Ho NF, et al. (1976) Systems approach to vaginal delivery of drugs II: In situ vaginal absorption of unbranched aliphatic alcohols. J Pharm Sci 65(11): 1574-1578.

\section{ISSN: 2574-1241}

DOI: 10.26717/BJSTR.2019.21.003576

Jacob McGee. Biomed J Sci \& Tech Res

(C) (P) This work is licensed under Creative BY Commons Attribution 4.0 License

Submission Link: https://biomedres.us/submit-manuscript.php
12. (2013) American College of Obstetricians and Gynecologists. Solutions for surgical preparation of the vagina. Committee Opinion No. 571. American College of Obstetricians and Gynecologists. Obstet Gynecol 122: $718-720$.

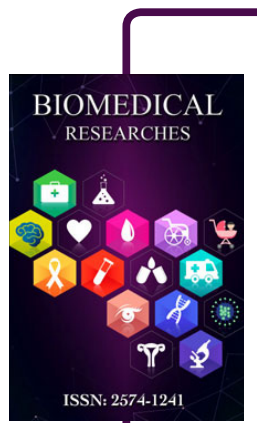

Assets of Publishing with us

- Global archiving of articles

- Immediate, unrestricted online access

- Rigorous Peer Review Process

- Authors Retain Copyrights

- Unique DOI for all articles

https://biomedres.us/ 